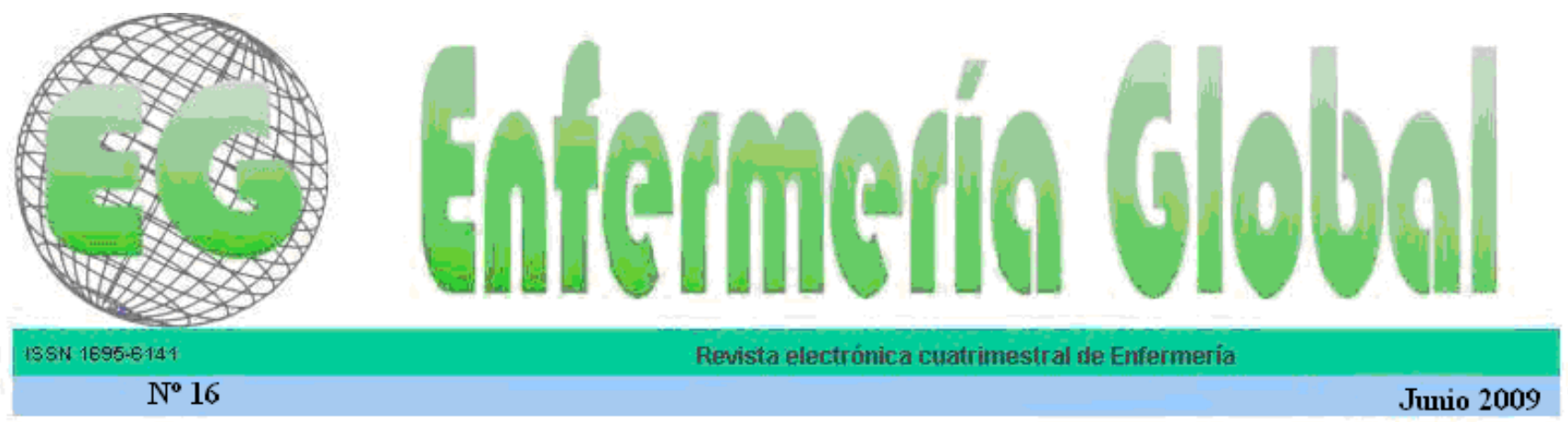

www.um.es/egloball

\title{
PROCESO DE MUERTE: EXPERIENCIA DE UN REGISTRO ENFERMERO COMO SOPORTE AL PROFESIONAL DE CUIDADOS PALIATIVOS
}

DEATH PROCESS: NURSING RECORD EXPERIENCE AS A SUPPORT OF THE PROFESSIONAL IN THE PALLIATIVE CARE UNIT

\author{
*Moreno Roldán, F., " Novellas Aguirre de Cárcer, A. \\ *Enfermero Servicio de Cuidados Paliativos. **Trabajadora Social. Coordinadora de la Unidad de Trabajo \\ Social. Instituto Catalán de Oncología. L' Hospitalet. Barcelona.
}

Palabras clave: Situación de últimos días. Cuidados Paliativos. Registros de Enfermería. Cuidados al final de la vida, Cáncer

Keywords: Last Days' Condition, Palliative Care Unit, Nursing Records, Care at the End of Life, Cancer.

\section{RESUMEN}

La situación de últimos días se caracteriza por la presencia de síntomas múltiples, multifactoriales y cambiantes, con alto impacto emocional, social y espiritual que precisa de atención específica del profesional en Cuidados Paliativos.

Desde el inicio de su funcionamiento como Unidad de Cuidados Paliativos en el Instituto Catalán de Oncología, se detectó la necesidad de tener una hoja de registro donde plasmar todo el proceso de la situación de agonía.

El objetivo de nuestro estudio es doble, por un lado describir la complejidad de la situación de últimos días que se refleja en los registros de Enfermería y cómo estos registros resultan de utilidad para el seguimiento posterior de duelo e intervención de otros profesionales, y por otro valorar de manera cualitativa su validez para los propios profesionales.

Este estudio descriptivo se diseñó en tres fases, con diferente metodología en cada una de ellas. En la primera fase, se analizaron los registros de la situación de últimos días de 150 enfermos durante 1 año que fallecieron en la unidad para detectar su necesidad de seguimiento del duelo. En la segunda fase se definen, en reuniones de consenso, los puntos que debe contener la hoja de registro que se va a utilizar y se diseñan sus cambios. En la tercera fase se evalúa de manera cualitativa la cumplimentación, satisfacción y utilidad como instrumento de registro específico.

Como resultante se detecta que una de cada cuatro situaciones de muerte recogidas en nuestra muestra son "procesos con complejidad", con alto impacto emocional para el equipo. $27 \%$ requirió seguimiento de duelo, bien por trabajadora social o por profesional de Psiquiatría en casos 
patológicos. Con respecto a la satisfacción del propio registro, los profesionales enfermeros valoran que sí resultaba útil, específico para esta situación y mejorable. La implicación en la práctica de estos resultados permite abordar mejor el proceso de últimos días, dictaminar situaciones complejas y elaborar unas actuaciones posteriores en el duelo.

\section{ABSTRACT}

Cancer patients' last days' condition has been described as multiple, multi-faceted and changing symptoms that mean high emotional, social and spiritual impact and requires specific attention from palliative care professionals.

Since the starting period of the Palliative Care Unit at the Institut Català of Oncology (ICO) in Barcelona, the necessity of a record sheet was discovered, in order to record the whole process of the agony condition.

The study has a double objective; on one hand to describe the complexity of last days' condition that is recorded in the nursing records and how these records are useful for the following-up of the mourning period and the intervention of other professionals. On the other hand, it asses, from a qualitative point of view, its validity for the professionals themselves.

This descriptive study was designed in three stages following a different methodology in each. During the first stage, records were analyzed of the condition of the last days of 150 patients that died in the unit over a year, so as to detect the need of following-up on mourning. In the second stage, by consensus in a meeting, the points that should be contained in the record sheet that were going to be used were defined and the modifications were written In the third stage, filling out, satisfaction and utility as an instrument of a specific record were assessed.

As a result, it was discovered that one of every four conditions of death registered in our sample were "complex processes" with a high emotional impact for the team. A percentage of $27 \%$ required mourning tracking by the social worker or a professional from the Psychiatry Department for pathologic cases. Regarding the satisfaction of the record itself, nursing professionals stated it was useful, particularly for this condition and that it could be improved. Being involved in the practice of these results allows us to better approach the process of the last days, to dictate complex conditions, and to prepare future actions for the mourning period.

\section{INTRODUCCIÓN}

Según los estándares de la Sociedad Española de Cuidados Paliativos ${ }^{(1)}$, estos consisten en la atención integral, individualizada y continuada a personas con enfermedad en situación avanzada y terminal así como a las personas a ellas vinculadas por razones familiares o de hecho. Esta situación de últimos días viene caracterizada por la presencia de síntomas múltiples, multifactoriales y cambiantes, con alto impacto emocional, social y espiritual que condicionan una elevada necesidad y demanda de atención. Esta demanda es atendida por los profesionales de forma holística, competente y con objetivos terapéuticos centrados en mejorar el confort y la calidad de vida, definidos según el propio enfermo y su familia, con respeto a su sistema de creencias, preferencias y valores.

De esta forma los instrumentos básicos deben ser los siguientes ${ }^{(2)}$ :

- Control de síntomas: Saber reconocer, evaluar y tratar adecuadamente los numerosos síntomas que aparecen e inciden directamente sobre el bienestar de los enfermos.

- Apoyo emocional y comunicación con el enfermo, familia y equipo terapéutico, estableciendo una relación franca y honesta. 
- Cambios de la organización que permitan el trabajo interdisciplinario y una adaptación flexible a los objetivos cambiantes de los enfermos.

- Equipo interdisciplinar, que disponga de espacios y tiempos específicos para el trabajo conjunto, además de una formación específica.

La puesta en común, el análisis y planificación de cada una de las situaciones tienen una dinámica imparable que permite al equipo sentirse respaldado por el resto de los profesionales.

Una forma que diferencia a los equipos de cuidados paliativos respecto a otros servicios de la medicina tradicional es su interés en crear una red de relación interdisciplinar donde los conocimientos de cada uno de los miembros implicados y el desarrollo de su disciplina vaya acorde a una toma de decisiones y un plan de actuación conjuntos. Estos objetivos terapéuticos, una vez elaborados y consensuados por el equipo interdisciplinar, han de cubrir y dar respuesta a las necesidades físicas, emocionales, sociales y espirituales del enfermo y familia ${ }^{(3)}$.

\section{El Servicio de Paliativos}

El Servicio de Cuidados Paliativos fue creado en 1995 dentro de la Zona de Hospitalización del Instituto Cátala de Oncología (ICO), en el Hospital Durán y Reynals en la ciudad de Barcelona.

Basándose en la filosofía de la Medicina Paliativa, donde lo que se pretende es dotar de máximo confort al enfermo y a su familia durante el proceso final de su enfermedad, es necesario contemplar las necesidades de manera global y holística.

Nuestro equipo multidisciplinar se organiza para cubrir las demandas y necesidades de enfermos y familiares en situaciones de enfermedades oncológicas avanzadas. La mortalidad en este servicio es cercana al $100 \%$, ello supone estar atendiendo situaciones de alta complejidad en cuanto a sintomatología e impacto emocional que se sufre. ${ }^{(3)}$

Enfermería, (se entiende como equipo directamente involucrados en los cuidados, y profesionales enfermeras y auxiliares), utiliza registros útiles tanto para el control de síntomas del enfermo durante su estancia como diversas hojas de valoración de necesidades, así como registros compartidos con los otros profesionales de la unidad (médicos, psicólogos, fisioterapeutas, Trabajo Social). El registro de la agonía y de los últimos días se fue modificando a lo largo del tiempo para, sin sobrecarga adicional al "quehacer" cotidiano, hacerla más ágil y funcional. Surgió como necesidad propia de las enfermeras para hacer operativa la comunicación interprofesional que hasta el momento se transfería de forma no sistemática en espacios informales. Los inicios de este instrumento se presentaron en el Congreso de la SEOM (Mallorca, 2001), con el título: "Vivencias de Enfermería a través de una hoja de registro en un hospital oncológico". Con un análisis preliminar de las vivencias enfermeras, sus puntos fuertes y áreas de mejora ${ }^{(4)}$.

\section{Descripción del circuito}

Semanalmente se lleva a cabo una revisión de las hojas de registro. Dos representantes del equipo, en este caso la Trabajadora Social, responsable del programa de duelo y un representante de Enfermería, revisan caso por caso y se deciden qué casos cumplen criterios de necesitar seguimiento más cercano y específico. Después de esta evaluación se 
lleva a cabo el envío de una carta de condolencia a la familia donde se les recuerda nuestra disponibilidad para su atención a través del programa de duelo y se les ofrece además la posibilidad de participar en los grupos de apoyo posterior.

Las hojas de registro permiten también al trabajador social, cuando concierta una entrevista de duelo, invitar a la Enfermería de referencia a participar en esta y recibir los agradecimientos, aclarar las dudas o compartir sentimientos en este marco terapéutico, dada la estrecha relación entre familia y equipo.

\section{OBJETIVO GENERAL}

1.- Describir cómo se recoge actualmente en el registro enfermero la "Situación de los últimos días" que permita entender las reacciones familiares durante el proceso y la posterior necesidad de seguimiento de duelo.

\section{OBJETIVOS ESPECÍFICOS}

1.-Actualizar qué puntos se deben contener en un nuevo formato de registro de la situación de últimos días.

2.- Recoger cómo refleja su propio impacto emocional el profesional de Enfermería.

3.- Recoger el nivel de satisfacción de los profesionales con el propio registro.

\section{MÉTODO}

\section{Tipo de diseño:}

Estudio retrospectivo - descriptivo sobre la implantación de un registro enfermero para la situación de los últimos días. El estudio comprende tres fases.

\section{Fases del estudio:}

\section{- Fase I:}

En esta primera fase se realiza un análisis de los 150 registros enfermeros sobre la situación de últimos días de enfermos que fallecieron durante 1 año en la unidad, para comprender cómo quedaba registrado por los profesionales que participaban en él.

Dichos registros habían sido completados por cada enfermero de referencia del paciente en el momento del fallecimiento, quedando la hoja cerrada con específica indicación de qué actividades de derivación se detectaban.

Se procedió, así mismo, al análisis de qué situaciones del paciente o familia indicaban que eran candidatas a seguimiento del duelo posterior y se detectaban en ese momento.

\section{- Fase II:}

Esta fase se centra en la definición de qué variables son importantes para ser registradas en la situación de últimos días. 


\section{VARIABLES CONTENIDAS EN EL REGISTRO UTILIZADO:}

- Cuidador (el personal de enfermería identifica al cuidador principal).

- Tiempo desde que está ingresado (Desde que ingresa hasta que es éxitus).

- Diagnóstico médico, tipo de tumor.

- Tiempo de Agonía (contabilizado en horas o días). Actualmente se utiliza la escala de Ramsay desde una puntuación de 5/6.

- Síntomas que han llevado a ingresar y destacan en los últimos días.

- Estado emocional del paciente (se define como negación, aceptación, regresión, sublimación y huida)

- Estado emocional del grupo familiar (si están adaptados al proceso, si hay conspiración de silencio, buen ajuste emocional o si hay protección informativa).

- Complicaciones que hayan podido surgir. (anotamos eventos tanto físicos, emocionales o sociales que se dan durante el proceso)

- Familiares presentes en el momento de la muerte.

- Relación con el equipo. (Se registran si han existido tensiones equipo-familia y el trabajo de soporte realizado con ella)

- Relación entre Familiares. (Problema entre sus miembros, se anota normalidad o dificultades)

- Propuesta inicial de seguimiento del duelo. (Si se perciben criterios de duelo patológico o no), ello conllevaba a una invitación posterior a las sesiones de duelo que se realizan en la institución.

- Percepción personal del profesional de Enfermería. ¿Cómo han vivenciado el proceso? Ha sido fácil, de alta implicación emocional, feed-back positivo con la familia o no.

- Observaciones / Imprevistos. (Aquí anotamos cualquier tipo de incidencia que haya dificultado el proceso asistencial.

- Registrado por... (Registro y firma del profesional enfermero que ha "vivido" la situación).

Tabla 1.- Puntos que contiene el registro enfermero elaborado.

\section{- Fase III:}

En esta fase se pidió a todas las enfermeras que trabajan en la Unidad de Cuidados Paliativos y a la Trabajadora Social que colabora en la valoración social de nuestros 
enfermos, que participaran en contestar un cuestionario para la medición del grado de satisfacción con respecto al propio registro utilizado durante todo un año.

El objetivo de tal medida fue conocer la utilidad descrita para los propios profesionales sobre el instrumento en sí o registro.

Se les pidió a todos los profesionales que utilizaban los registros de manera diaria y que participaban en el proceso asistencial de los enfermos en situación de últimos días su colaboración.

Se utilizó un cuestionario elaborado con 3 preguntas abiertas no estructuradas que valoraban las siguientes dimensiones:

1.- ¿Qué crees que te aporta cumplimentar la hoja?

2.- ¿Crees que es un instrumento válido para tu trabajo, te ayuda en el día a día?,

3.- ¿Añadirías alguna cosa?

El análisis de los datos atendió tanto a las variables cuantitativas en términos de frecuencias y porcentajes, como a las de tipo cualitativo con el uso específico de técnicas de análisis de contenido textual.

\section{RESULTADOS:}

De la muestra total de Historias y registros analizados $(\mathrm{N}=150)$, que describían los procesos de muerte que han sucedido en la unidad, los resultados en cuanto al ajuste emocional que los enfermeros describieron en ellos, la distribución de frecuencias fue:

$\checkmark \quad$ Un $74 \%$ de la muestra quedaron registrados como Procesos sin complejidad, que describían el buen ajuste a la situación, con adecuado control de síntomas, adaptación al proceso tanto del paciente como de la familia. Los registros enfermeros describían en estos casos frases como: "Buen control de síntomas, familia ajustada....".

$\checkmark \quad 23 \%$ fueron clasificadas como Procesos con complejidad, quiere decir que presentaban descontrol de síntomas, que se daba en personas jóvenes con procesos rápidos sin tiempo de intervención por parte del equipo; (muerte súbita o inesperada vs. anunciada), crisis de pánico en pacientes o familia. Las enfermeras registraron: "Impacto emocional familiar. Las emociones son transmitidas al equipo, .....personalmente lo he vivido de forma muy angustiante por la carga sentimental del caso."

$\checkmark \quad$ Sólo el 3\% de situaciones se clasificaron como Procesos de alta complejidad ya que presentaron alto impacto emocional frente a la situación o fallecimiento en soledad que supuso mayor carga emocional y gran dificultad añadida a los profesionales presentes. "Muere sólo,..... ver morir a alguien sólo es difícil de digerir". 


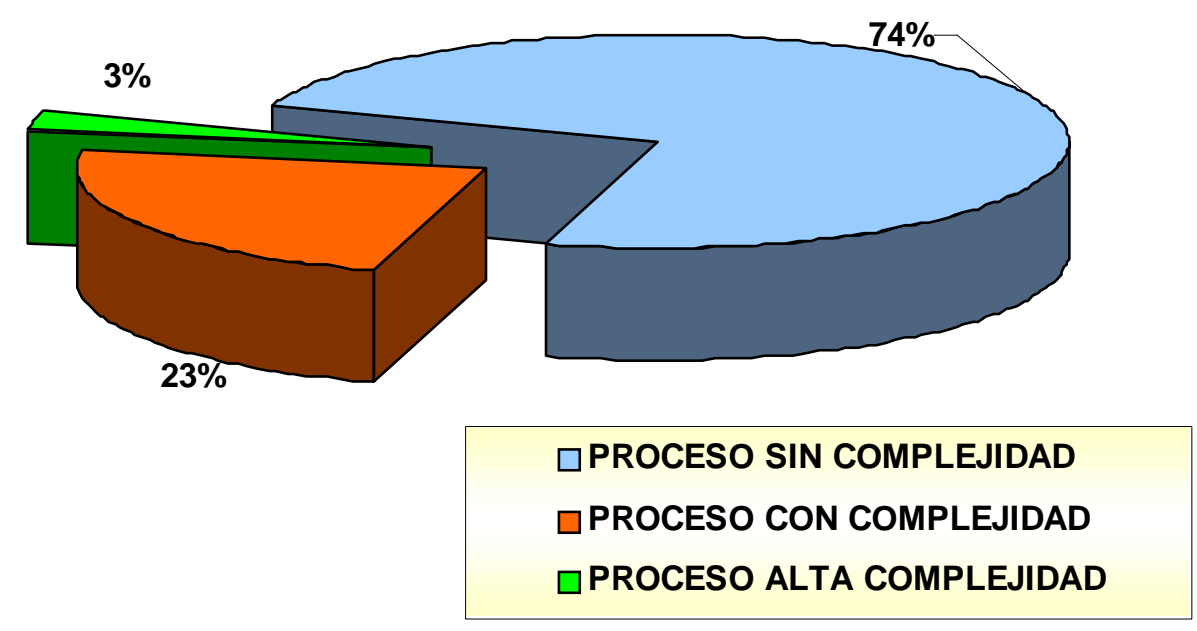

Gráfico 1.- Distribución de la muestra en cuanto a su complejidad en el proceso de la muerte

En cuanto a qué seguimiento de duelos que se habían generado a raíz de la valoración de los registros enfermeros las categorías se agruparon en los siguientes grupos:

\begin{tabular}{|c|l|}
\hline $32 \%$ & Procesos de duelo adecuados y de resolución favorable \\
\hline $27 \%$ & $\begin{array}{l}\text { Procesos de duelo que necesitaron apoyo específico, pero su } \\
\text { resolución fue favorable }\end{array}$ \\
\hline $25 \%$ & $\begin{array}{l}\text { Procesos que tras un periodo de seguimiento la familia decide no } \\
\text { seguir con las visitas de duelo. }\end{array}$ \\
\hline $2 \%$ & $\begin{array}{l}\text { Procesos que precisan una intervención específica Psiquiátrica y } \\
\text { derivación para control en el centro de Salud Mental de } \\
\text { referencia. }\end{array}$ \\
\hline
\end{tabular}

Tabla 2.- Procesos detectados que precisaron seguimiento de duelo.

\section{ANÁLISIS CUALITATIVO DEL CONTENIDO DE LAS VIVENCIAS ENFERMERAS}

\section{Resultados de la Fase III:}

Se valoraron las preguntas de manera cualitativa, siguiendo las directrices del análisis de contenido.

Algunas frases nos parecen representativas de los aspectos cualitativos de la situación. 
Se describen situaciones de fuerte contraste como las siguientes:

“.........ha descargado.... (refiriéndose a la familia en el momento de la muerte) una tempestad contra todo y todos, se desbordó toda la contención que había tenido hasta ese momento....".

“....Es realmente emocionante el vínculo existente en esta familia, es un caso difícil de olvidar...".

Agrupando los resultados de las vivencias con respecto al proceso de la situación de últimos días podemos dividirlas, atendiendo a su significado positivo o negativo en dos grandes grupos:

\section{VALORACIÓN CON SIGNIFICADO POSITIVO}

Ordenados de mayor a menor frecuencia ${ }^{(\mathrm{N})}$ entre los participantes según sus citas

\begin{tabular}{|c|c|}
\hline $\begin{array}{l}\text { REFLEXIÓN } \\
\text { PERSONAL }\end{array}$ & "Sí,.... me ayuda a reflexionar en el tema de la muerte......" \\
\hline $\begin{array}{l}\text { REGISTRO COMO } \\
\text { ESPACIO ÚTIL } \\
\text { COMO } \\
\text { PROFESIONALES }\end{array}$ & $\begin{array}{l}\text { "Espacio de síntesis, medio donde poder plasmar......" "Poder } \\
\text { seguir una sistemática de recogida de datos......" "Al revisar o } \\
\text { pensar en cosas concretas me facilita el trabajo...." } \\
\text { "... es funcional y con un vistazo tienes un buen perfil de la } \\
\text { situación" }\end{array}$ \\
\hline $\begin{array}{l}\text { COMO EXPRESIÓN } \\
\text { EMOCIONAL }\end{array}$ & $\begin{array}{l}\text { "Válvula de escape...", "permite ventilar emociones", ...." Todo } \\
\text { lo que tú sientes en ese momento se te permite plasmarlo en un } \\
\text { papel ". "Me ayuda a poder canalizar mis emociones...." }\end{array}$ \\
\hline $\begin{array}{l}\text { APORTACIÓN } \\
\text { PROFESIONAL }\end{array}$ & $\begin{array}{l}\text { "Recoger datos para estudios......., "es un buen instrumento para } \\
\text { (recoger) las habilidades de atención y sensibilidad de mis } \\
\text { compañeros profesionales, con respecto a las familias , al } \\
\text { enfermo y al proceso" }\end{array}$ \\
\hline $\begin{array}{l}\text { COMO CIERRE DE } \\
\text { PROCESO, } \\
\text { DESPEDIDA }\end{array}$ & $\begin{array}{l}\text { "Para mí es una forma de despedida de la persona fallecida y de } \\
\text { su familia" }\end{array}$ \\
\hline
\end{tabular}

Tabla 3.- Valoraciones con significado positivo. 
Como valoración de resultado podemos reseñar que los profesionales consideran importante tener un espacio para poder discutir sobre la muerte y cómo ello ha repercutido en sí mismos

\section{VALORACIÓN CON SIGNIFICADO NEGATIVO}

Según frecuencia de referencias entre los participantes

\begin{tabular}{|c|c|}
\hline $\begin{array}{l}\text { EL REGISTRO } \\
\text { NECESITA } \\
\text { MODIFICACIONES }\end{array}$ & $\begin{array}{l}\text { "Necesito más espacio (en referencia a espacio para escritura) } \\
\text { en los ítems psico-emocionales, familia....", } \\
\text { "Propuesta de anonimato.....", "Creo que es un área de mejora.." }\end{array}$ \\
\hline $\begin{array}{l}\text { NO DEVOLUCIÓN DE } \\
\text { RESULTADOS }\end{array}$ & $\begin{array}{l}\text { "no tengo feed-back, es decir no tengo los resultados sobre las } \\
\text { familias de mis cuidados-atenciones", } \\
\text { "si se hace para valorar como ha ido cada proceso de éxitus...." }\end{array}$ \\
\hline $\begin{array}{l}\text { INSUFICIENTE } \\
\text { TRABAJO DE } \\
\text { EQUIPO }\end{array}$ & $\begin{array}{l}\text { ".....Se podría sacar mayor partido, si utilizáramos la hoja (el } \\
\text { registro), para comentarlo posteriormente con el resto del } \\
\text { equipo". } \\
\text { "herramienta cotidiana para beneficio propio, pero principalmente } \\
\text { pensado en el resto del equipo que pudiese utilizarlo..." }\end{array}$ \\
\hline NO SE UTILIZA & $\begin{array}{l}\text { "Sinceramente en el día a día no la utilizo, una vez la relleno no } \\
\text { vuelvo a revisar nada" } \\
\text { "No siempre se dispone de tiempo para rellenarlo... creo que es } \\
\text { una pena, porque es el momento ideal cuando acaba de } \\
\text { ocurrir....." }\end{array}$ \\
\hline $\begin{array}{l}\text { PREFIEREN OTROS } \\
\text { FORMATOS }\end{array}$ & $\begin{array}{l}\text { "Es un buen instrumento, pero para expresar mis sentimientos } \\
\text { prefiero hablar con un compañero antes que plasmarlo en una } \\
\text { hoja ". }\end{array}$ \\
\hline
\end{tabular}

Tabla 4.-Valoraciones con significado negativo.

\section{DISCUSIÓN}

En la bibliografía se han descrito las actitudes del enfermero ante el paciente terminal. Hay estudios como el de Molina J.M. en 2006, que miden la satisfacción a cuidadores en una Unidad de Cuidados Paliativos. Constan en la literatura, Rich S \& Worden J W, datos sobre 
actitudes en la situación de últimos días de pacientes y familias. Nuestro estudio reflexiona sobre aspectos que otros autores no habían abordado hasta ahora, nosotros exploramos sobre las propias actitudes de los profesionales enfermeros con respecto al proceso de muerte. Así mismo, tampoco existen análisis de la eficacia de los registros que se utilizan en las unidades.

Como limitaciones al estudio en cuanto a su realización, se puede resaltar que se ha llevado a cabo en un ámbito local circunscrito a una sola unidad de Cuidados Paliativos y aunque se compone de un amplio equipo de 30 personas la generalización de los datos no será posible dada la diferencia de equipos y de contextos.

\section{CONCLUSIONES}

- Se considera que la hoja de registro utilizada es un instrumento funcional que permite reflejar cómo ha ido el proceso, así como recoger las valoraciones enfermeras de la situación de últimos días.

- $\quad$ Permite una planificación de acciones e intervenciones anticipadas relacionadas con la muerte. Tanto para las familias como para los profesionales, mediante reuniones interdisciplinarias con el resto del equipo, facilitar en aquellos procesos difíciles, intercambiar la información de cómo fue la muerte. Es especialmente valiosa en las visitas de duelo posterior.

- $\quad$ Según nuestro estudio, uno de cada cuatro pacientes presentaron proceso de muerte con grado de complejidad que requiere especial atención por parte del equipo y un registro donde dejar constancia.

- $\quad$ Tener un registro de este tipo en una Unidad de Cuidados Paliativos es útil según resulta de la valoración que los profesionales realizaron en la encuesta de satisfacción.

- Permitir un espacio de reflexión, donde dejar escrito las vivencias y emociones resulta ser un excelente final para "cerrar un proceso de vida".

\section{AGRADECIMIENTO}

- Agradecemos la inestimable ayuda de Ma Paz Fernández Ortega por su asesoría.

\section{BIBLIOGRAFÍA:}

1.- Gómez-Batiste X, de la Mata I, Fernández M, Ferrer JM, García E, Novellas A., et alt. Grupo de Calidad de la SECPAL. Guía de Criterios de Calidad en Cuidados Paliativos. Planificación Sanitaria. Editado Ministerio de Sanidad y Consumo. 2002; 7-8

2.- Gómez-Batiste X, Planas J, Roca, J y Viladiu P. Cuidados Paliativos en Oncología. 1르 Ed. Barcelona: JIMS; 1996. p 1-3 definiciones.

3.- Cuidados Paliativos. Recomendaciones de la SECPAL (pg19). Edita Ministerio de Sanidad y Consumo 1993. 
4.- Moreno F. Vivencia de Enfermería en un servicio de Cuidados Paliativos (SCP) de un hospital oncológico. Libro de resúmenes. Comunicación presentada en el congreso Nacional Enfermería Oncológica de la SEEO. Mayo 2001

5.- Grupo de trabajo de la Comisión de Mortalidad de CSUB(Ciudad Sanitaria y Universitaria de Bellvitge), Protocolo de agonía. Julio 1998. Pág 19-23

6.- Corrales E. Reflexiones sobre la Enfermería en Cuidados Paliativos. Med. Pal. 2004;(3); 133-134.

7.- Godoy Fernández MD, Garcia Puche JL et alt. Efectos de los Cuidados Paliativos en la Calidad de Vida de paciente oncológicos avanzados. Medicina Paliativa 1999; 6(4): 131-132

8.-Lacasta MA, Kroeff $P$, Sastre $P$, Casero A, Martinez AM. Una intervención en grupo para personas en duelo. Med. Pal. 2000;(7);1:6-9.

9.- Novellas A: La atención a las familias en cuidados paliativos. Análisis del Proceso y propuestas metodológicas de intervención. Med. Pal. 2000; 7 (2): 49-56.

10.- Rich S. Ayudar a las familias a afrontar la muerte y la agonía. Nursing, 1997:29-34

11.-Tomás Sábado J .La medida de la ansiedad ante la muerte en profesionales de enfermería. .Enfermería Científica 2002;246-247;42-7

12.- Torres Prieto, M. I. Reflexión sobre la actuación enfermera ante los pacientes terminales; Metas de Enfermería $2001 ; 36: 52-54$

13.- Sanz de la Torre J C, De la Peña E, Goméz Galán R, Garrido J, Herrero A. Actitudes del enfermero-a ante el paciente terminal. Enfermería Científica 2000; 220-221: 17-20 14.- Sanz OJ, Gómez Batiste AX, Gómez SM, Núñez OJ. Cuidados Paliativos. Recomendaciones de la Sociedad Española de Cuidados Paliativos. Madrid: Guías y Manuales, Ministerio de Sanidad y Consumo, 1993.

15.- White, Kenneth R., Coyne, Patrick J. \& Patel, Urvashi B. Are Nurses Adequately Prepared for End-of-Life Care?. Journal of Nursing Scholarship. 2001; 33 (2), 147-151.

16.- Worden J W. El tratamiento del duelo. Asesoramiento psicológico y terapia Madrid : Paidós; 1997.

17.- Molina JM, Romero J, Romero Y, Rodríguez RM, Gálvez R. Med. Pal. Vol13: º1 ;11-17, 2006.

ISSN 1695-6141

(C) COPYRIGHT Servicio de Publicaciones - Universidad de Murcia 